\title{
Concentración de nitrato, fosfato, boro y cloruro en el agua del río Lerma
}

\section{Concentration of nitrate, phosphate, boron and chloride in the Lerma River}

\author{
José Pedro Pérez-Díaz ${ }^{1 *}$ ㅇ, Héctor Manuel Ortega-Escobar ${ }^{1}$ 이, Carlos Ramírez-Ayala1 0 , Héctor

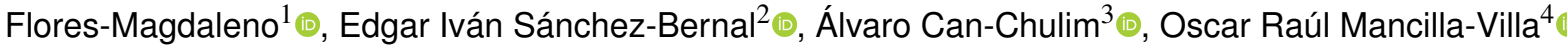 \\ ${ }^{1}$ Posgrado de Hidrociencias, Colegio de Postgraduados. Carr. México-Texcoco Km. 36.5. CP. 56230. Montecillo Texcoco, Estado de \\ México, México. \\ ${ }^{2}$ Departamento de Ecología, Universidad del Mar. Ciudad Universitaria, CP. 70902. Puerto Ángel, Pochutla, Oaxaca, México. \\ ${ }^{3}$ Unidad Académica de Agricultura, Universidad Autónoma de Nayarit. Carr. Tepic-Compostela Km. 9, CP. 63155. Xalisco, Nayarit, \\ México. \\ ${ }^{4}$ Departamento de Producción Agrícola, Centro Universitario de la Costa Sur, Universidad de Guadalajara. Av. Independencia Nacional \\ \#151, CP. 48900. Autlán de Navarro, Jalisco, México. \\ *Autor de correspondencia: josepedro.perez@colpos.mx
}

Nota científica recibida: 08 de mayo de 2018 aceptada: 06 de septiembre de 2018

RESUMEN. El río Lerma se origina en la laguna de Almoloya, Estado de México y desemboca en el Lago de Chapala, Jalisco. El objetivo fue conocer la concentración de nitrato $\left(\mathrm{NO}_{3}^{-}\right)$, fosfato $\left(\mathrm{PO}_{4}^{3-}\right)$, boro $\left(\mathrm{B}^{3+}\right)$ y cloruro $\left(\mathrm{Cl}^{-}\right)$en el río Lerma para estimar el riesgo de toxicidad de algunos cultivos irrigados con sus aguas. El promedio de concentración para el $\mathrm{NO}_{3-}$ fue de $27.48 \mathrm{mg} \mathrm{L}^{-1}$, para $\mathrm{PO}_{4}^{3-}$ de $4.027 \mathrm{mg} \mathrm{L}^{-1}$, para B ${ }^{3+}$ de $0.496 \mathrm{mg} \mathrm{L}^{-1}$ y para $\mathrm{Cl}^{-}$de $1.974 \mathrm{meq} \mathrm{L}^{-1}$. El coeficiente de variación indica alta heterogeneidad en la concentración iónica del agua, atribuida a las descargas de agua residual doméstica, industrial y drenaje agrícola. El río Lerma tiene diferente grado de restricción de uso con respecto al riesgo de toxicidad por iones específicos.

Palabras clave: Calidad del agua, riego agrícola, toxicidad por iones específicos.

ABSTRACT. The Lerma River originates in the Almoloya Lagoon, State of Mexico and empties into Lake Chapala, Jalisco. The objective of this study was to determine the concentration of nitrate $\left(\mathrm{NO}_{3^{-}}\right)$, phosphate $\left(\mathrm{PO}_{4}^{3-}\right)$, boron $\left(\mathrm{B}^{3+}\right)$ and chloride $\left(\mathrm{Cl}^{-}\right)$in the Lerma River to estimate the risk of toxicity of some crops irrigated with its waters. The average concentration was $27.48 \mathrm{mg} \mathrm{L}^{-1}$ for $\mathrm{NO}_{3^{-}}, 4.027 \mathrm{mg} \mathrm{L}^{-1}$ for $\mathrm{PO}_{4}^{3-}, 0.496 \mathrm{mg} \mathrm{L}^{-1}$ for $\mathrm{B}^{3+}$ and 1.974 meq $\mathrm{L}^{-1}$ for $\mathrm{Cl}^{-}$. The coefficient of variation indicates high heterogeneity in the ionic concentration of the water, attributed to discharges of domestic, industrial and agricultural drainage wastewater. The Lerma River has a different degree of restriction of use with respect to the risk of specific ion toxicity.

Key words: Water quality, agricultural irrigation, specific ion toxicity.

\section{INTRODUCCIÓN}

El río Lerma forma parte de la cuenca LermaSantiago-Pacífico. Debido a que los manantiales que le daban origen en la laguna de Almoloya, han desaparecido, actualmente solo recibe agua en la época de lluvias. El río tiene su origen en un canal formado por una serie de ciénegas del sistema lacustre de la laguna de Almoloya a $2588 \mathrm{msnm}$ y desemboca en el lago de Chapala, en el Estado de Jalisco a 1530 msnm (Cotler-Avalos et al. 2006). Sus aguas tienen uso doméstico, agrícola, pecuario, industrial y para la generación de energía eléctrica (Zarazúa et al. 2013). En su curso, recibe aguas de corrientes tributarias de Ios ríos: Laja, Guanajuato, Turbio, Tigre y Duero; y en su cauce se encuentran las presas Antonio Alzate, Tepuxtepec y Solís, que se utilizan para riego agrícola y generación de energía eléctrica (Cotler-Avalos et al. 2006).

Con base en el índice de calidad del agua (ICA), el rio Lerma presenta las siguientes condiciones de calidad: altamente contaminado, poco con- 
taminado y aceptable, por lo que se recomienda su uso para la agricultura (López-Hernández et al. 2007). Al respecto Zamora et al. (2008) y Belaid et al. (2012) indican que las aguas residuales vertidas al río contienen nitrógeno y fósforo, por lo que sus aguas pueden influir de forma positiva en el rendimiento de los cultivos. Pero también representan un foco de infección para la salud humana (Cifuentes et al. 2000), y la toxicidad del ambiente (Robledo-Zacarías et al. 2017).

La alta concentración de nitrato y fosfato puede atribuirse a la aplicación excesiva de fertilizantes (Chávez-Alcántar et al. 2011), lo que provoca lixiviados y contaminación del agua subterránea, además de inducir eutrofización de los cuerpos de agua (Figueruelo-Alejano y Marino-Dávila 2004). Sobre lo mismo Ayers y Westcot (1987) indican que los problemas de calidad del agua están relacionados con su uso, desde la perspectiva agronómica, la concentración iónica de la solución afecta los cultivos debido al tipo de iones presentes (Sánchez-Bernal et al. 2008, Can-Chulim et al. 2014).

En la cuenca del río Lerma los principales cultivos son el sorgo, cebada, maíz, frijol, trigo, alfalfa, espárrago y brócoli (CONAGUA 2015), por lo que la irrigación con sus aguas puede afectar la germinación y el desarrollo (Can-Chulim et al. 2017). Debido a que el agua del río es bicarbonatada-sódica y bicarbonatada mixta con riesgo medio de salinidad (PérezDíaz et al. 2015), lo que puede ocasionar efectos negativos de salinidad en los cultivos y sodicidad en los suelos arcillosos (López-García et al. 2016). Los problemas de toxicidad por iones específicos se presentan en los cultivos con iones de $\mathrm{B}^{3+}, \mathrm{Cl}^{-}$y Na${ }^{+}$, que son absorbidos y acumulados en los tejidos de las plantas en concentraciones que provocan daño y reducen el rendimiento (Ayers y Westcot 1987), aunque la toxicidad depende de la tolerancia del cultivo (Sánchez-Bernal et al. 2013, Can-Chulim et al. 2017).

En lo referente al boro, la toxicidad se presenta en concentraciones entre 1 y $2 \mathrm{mg} \mathrm{L}^{-1}$ en el agua de riego (Mancilla-Villa et al. 2014, Maas 1990), por medio de manchas amarillas en el borde y ápice de las hojas. Por otro lado, cuando el agua contiene concentraciones de $\mathrm{Cl}^{-}$mayores de 4 meq $\mathrm{L}^{-1}$, se tienen restricciones de uso, ya que puede acumularse en las hojas y producir clorosis afectando la fotosíntesis (Ayers y Westcot 1987). Por lo anterior, el objetivo fue determinar la concentración de nitrato, fosfato, boro y cloruro en el agua del río Lerma, y estimar el riesgo de toxicidad por iones específicos $\left(\mathrm{B}^{3+} \mathrm{y} \mathrm{Cl}^{-}\right)$ que pueden afectar el desarrollo de los cultivos.

\section{MATERIALES Y MÉTODOS}

\section{Muestreo, análisis de agua y análisis estadístico}

Se colectaron y analizaron muestras de agua de 39 estaciones de muestreo distribuidas en el cauce del río Lerma (Figura 1). Las muestras se colectaron en abril de 2015 de acuerdo con la metodología de muestreo propuesta por la SECOFI (1980), teniendo en cuenta la accesibilidad a los sitios. La concentración de $\mathrm{NO}_{3^{-}}, \mathrm{PO}_{4}^{3-}$ y $\mathrm{B}^{3+}$ se determinó por espectrofotometría (JENWAY® 7305 Spectrophotometer), con longitudes de onda de 410, 880 y $420 \mathrm{~nm}$, respectivamente. $\mathrm{El} \mathrm{Cl}^{-}$se determinó por titulación volumétrica (Richards et al. 1982) con nitrato de plata $(0.01 \mathrm{~N})$; el $\mathrm{NO}_{3}$ - por el método de nitración del ácido salicílico (Robarge et al. 1983), el $\mathrm{PO}_{4}^{3-}$ mediante el método de ácido ascórbico (Eaton et al. 1998), y el $\mathrm{B}^{3+}$ por el método de la H-Azometina (Rodier 1978). Se utilizaron soluciones estándar de concentración conocida preparadas en el laboratorio para establecer las rectas de calibración con valor en la ordenada al origen de cero. La ecuación de regresión utilizada para estimar la concentración de nitrato $\left(\mathrm{NO}_{3^{-}}\right)$fue $\mathrm{NO}_{3^{-}}=(205.69 \times \mathrm{A})$ con $\mathrm{R}^{2}=0.98$. Para determinar la concentración de 0 a $3 \mathrm{mg} \mathrm{L}^{-1}$ de fosfato $\left(\mathrm{PO}_{4}^{3-}\right)$ se utilizó la ecuación $\mathrm{PO}_{4}^{3-}=(4.9147 \times \mathrm{A})$ con $R^{2}=0.99$, para la concentración de 3 a $15 \mathrm{mg}$ $\mathrm{L}^{-1}$ de fosfato $\left(\mathrm{PO}_{4}^{3-}\right)$ la ecuación fue $\mathrm{PO}_{4}^{3-}=(13.17$ $\times A)$ - 5.286 con $R^{2}=0.99$, y la ecuación para estimar la concentración de boro $\left(\mathrm{B}^{3+}\right)$ fue $\mathrm{B}^{3+}=(6.744 \times \mathrm{A})$ con $\mathrm{R}^{2}=0.99$.

El enfoque de la investigación fue de tipo cuantitativo con alcance descriptivo, la superficie irrigada con el agua del río Lerma se destina al cultivo de sorgo, cebada, maíz, frijol, trigo, alfalfa, espárrago y brócoli, por lo que la concentración iónica del agua 


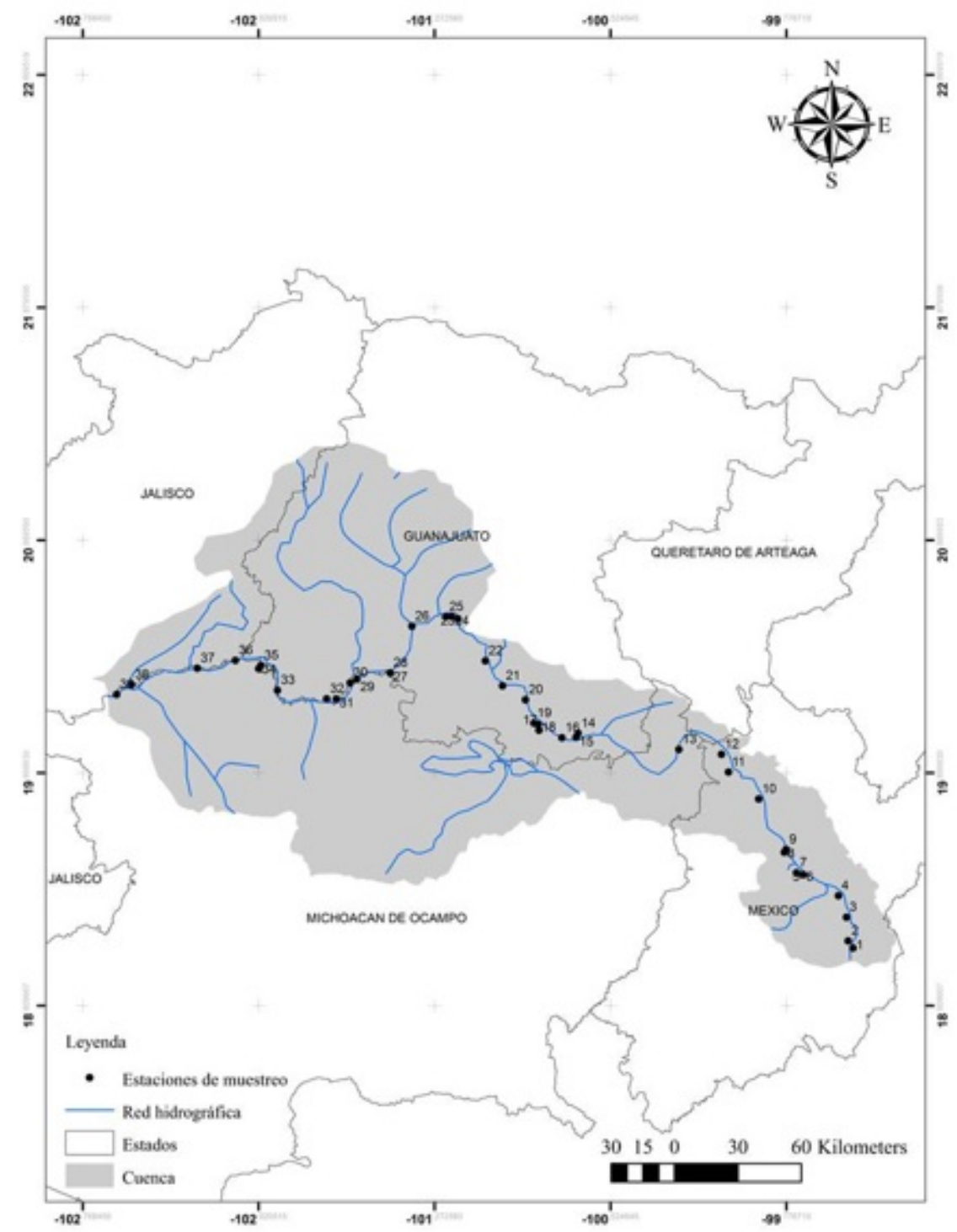

Figura 1. Localización del área de estudio.

puede afectar los cultivos (Maas 1990). Se determinaron los valores: mínimos, máximos, promedios, medianas, desviaciones estándar, amplitud, coeficientes de variación (CV), cuartiles y valores extremos (Montgomery y Runger 2015). Los análisis se realizaron con el software $\mathrm{SAS}^{\circledR}{ }^{\circledR}$ versión 9.0 y las gráficas con el programa SigmaPlot ${ }^{\circledR}$ versión 10.0.

\section{RESULTADOS Y DISCUSIÓN}

La cuenca del río Lerma recibe aguas residuales provenientes del uso doméstico, industrial y agrícola, por lo que contiene concentraciones de nitrógeno, fosforo, potasio, cobre, hierro y zinc. Al respecto, Duncan y Cairncross (1990) indican que estas aguas sirven para el uso agrícola, debido a que pueden reducir la necesidad de fertilizantes. Aunque la alta concentración de nitrato (Figura 2) y fosfato 


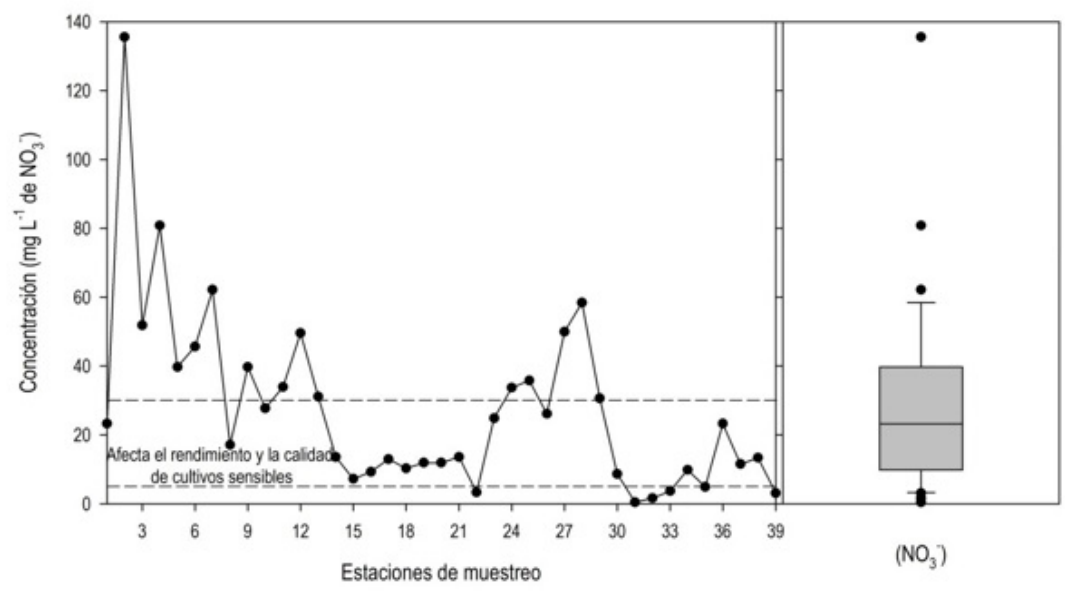

Figura 2. Distribución de la concentración de nitrato en el agua del río Lerma.

(Figura 3) es un indicador del estado de eutrofización del agua del río Lerma, los valores de $0.768 \mathrm{mg} \mathrm{L}^{-1}$ de $\mathrm{P}\left(2.35 \mathrm{mg} \mathrm{L}^{-1}\right.$ de $\left.\mathrm{PO}_{4}^{3-}\right)$ indican estado hipereutrófico. Al respecto, Moreno-Franco et al. (2010) y Zarazúa et al. (2013) reportan que concentraciones entre $0.384 \mathrm{mg} \mathrm{L}^{-1}\left(1.177 \mathrm{mg} \mathrm{L}^{-1}\right.$ de $\left.\mathrm{PO}_{4}^{3-}\right)$ y $0.096 \mathrm{mg} \mathrm{L}^{-1}$ de $\mathrm{P}\left(0.294 \mathrm{mg} \mathrm{L}^{-1}\right.$ de $\left.\mathrm{PO}_{4}^{3-}\right)$ lo que indican condiciones eutróficas, por lo que valores mayores de $2.35 \mathrm{mg} \mathrm{L}^{-1}$ de $\mathrm{PO}_{4}^{3-}$ del $69 \%$ de las estaciones de muestreo, indican condición hipereutrófica en la mayor parte del curso alto del río Lerma de Almoloya a la presa Tepuxtepec. Mientras que el estado hipereutrófico se detectó desde Salamanca hasta La Calle y de la vía Rivera a la Barca-lbarra; en tanto que el $31 \%$ de las estaciones de muestreo en el curso medio y bajo tuvieron condiciones eutróficas, por lo que el agua del río Lerma puede considerarse en mayor proporción hipereutrófico y en menor grado eutrófico. Al respecto Velázquez-Machuca et al. (2010) reportan que el río Duero, cuerpo receptor de agua residual y drenaje agrícola, tiene agua mixta-bicarbonatada y bicarbonatada-sódica clasificada como eutrófica. Además de la adición de boro por el vertido de aguas residuales (Velázquez et al. 2011). Lo cual puede influir en la concentración y composición iónica, debido a que el río Duero es un afluente del río Lerma. Al respecto Chavez-Alcantar et al. (2011) concluyen que los drenajes agrícolas y las aguas residuales son la principal fuente de contaminantes en la zona de la ciénega del Chapala. Debido a que las aguas residuales transportadas por el río Lerma desembocan en el Lago de Chapala, lo que influye en la concentración de contaminantes encontrados en este cuerpo de agua. En la zona del alto relieve (Toluca-Temascalcingo) son escasas las corrientes tributarias del río Lerma, por lo que la alta concentración a su paso por las principales ciudades, se debe al vertido de agua residual; en el curso medio (Acámbaro-Salvatierra-Jaral del Progreso), con tendencia a disminuir la concentración de nitrato. Pero en la vía Salamanca-Pueblo NuevoLabor de Peralta se incrementó, en el curso bajo del río Lerma (Pastor Ortiz-La Piedad-La Barca) la tendencia fue a disminuir la concentración de nitrato. La aplicación excesiva y pérdida de fertilizantes nitrogenados puede contribuir en la concentración de nitrato en los cuerpos de agua (Velázquez-Machuca et al. 2010).

En el curso medio y bajo del río Lerma, el agua se diluye por el aporte de diversas corrientes tributarias con baja concentración iónica, lo que contribuye con la depuración del río, con variación de 9.87 a $39.70 \mathrm{mg} \mathrm{L}^{-1}$ para la concentración de nitrato, y de 1.73 a $5.26 \mathrm{mg} \mathrm{L}^{-1}$ para el fosfato, en el $50 \%$ de las estaciones de muestreo del río Lerma. Lo que puede deberse a que el río Lerma se encuentra en 


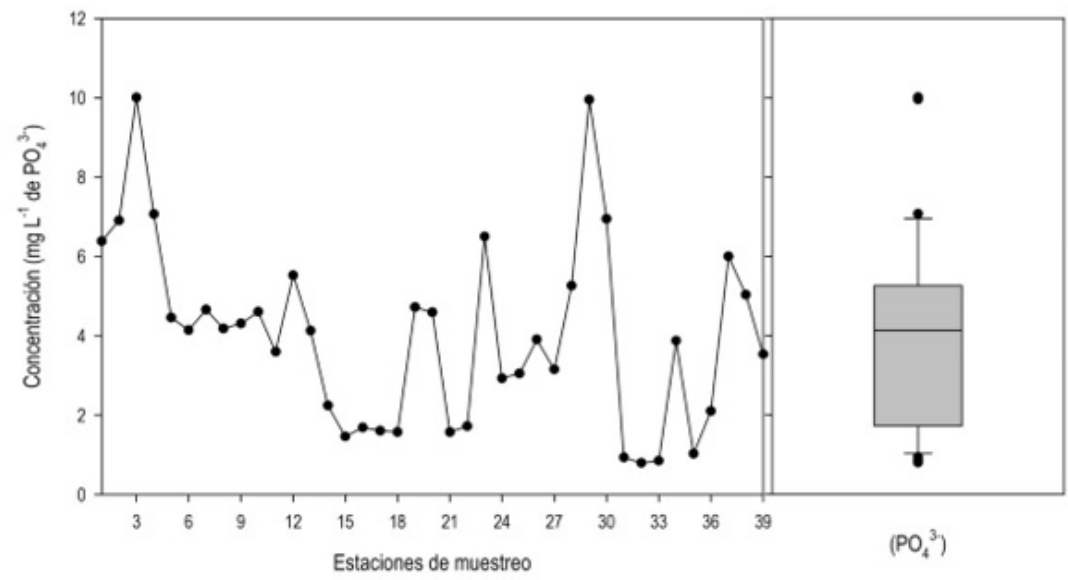

Figura 3. Distribución de la concentración de fosfato en el agua del río Lerma.

una zona de alta actividad agrícola e industrial, debido a la descarga de aguas residuales y al gradiente altitudinal de 2588 a $1530 \mathrm{msnm}$, lo que favorece su evacuación por medio de la corriente principal de la cuenca.

La principal fuente de fósforo en el agua residual puede deberse a los detergentes provenientes de actividades antrópicas que forman las aguas residuales domésticas e industriales, ya que algunos detergentes contienen fosfato de sodio. Así como el drenaje agrícola, por el lavado de fertilizantes fosfatados en áreas agrícolas (Velázquez-Machuca et al. 2010). A este respecto, Ayers y Westcot (1987) indican que el agua de riego con calidad aceptable generalmente debe contener menos de $2 \mathrm{mg} \mathrm{L}^{-1}$ de fosfato $\left(0.652 \mathrm{mg} \mathrm{L}^{-1}\right.$ de $\mathrm{P}$ ), mientras que Hem (1985) indica que la concentración en exceso es una fuente de nutrientes que contribuye con el desarrollo de algas y vegetación acuática, lo que puede ocasionar su acumulación y obstrucción de los sistemas de riego.

En las aguas naturales, el contenido de boro $\left(\mathrm{B}^{3+}\right)$ proviene del intemperismo químico de las rocas, del agua de regiones volcánicas y de fuentes hidrotermales. Además de que el tetraborato de sodio $\left(\mathrm{Na}_{2} \mathrm{~B}_{4} \mathrm{O}_{7} \cdot{ }_{10 \mathrm{H}_{2} \mathrm{O}}\right)$, se utiliza como agente limpiador, también tiene usos industriales, por lo que el boro puede estar presente en el agua de origen residual doméstica e industrial (Hem 1985, Velázquez et al. 2011). Aunque este ión es importante en la producción agrícola, en cantidades mayores de $1 \mathrm{mg}$ $\mathrm{L}^{-1}$ es tóxico para cultivos como los cítricos, frutales, frijol, brócoli y trigo (Page et al. 1990, Mancilla-Villa et al. 2014), mientras que los cultivos tolerantes como el sorgo y esparrago, no muestran síntomas en concentraciones de $4 \mathrm{mg} \mathrm{L}^{-1}$ de $\mathrm{B}^{3+}$ en la solución del suelo (Pratt y Suarez 1990). Para una misma concentración de $\mathrm{B}^{3+}$ en el agua de riego, se tiene diferente concentración de $\mathrm{B}^{3+}$ en la zona de raíz, lo que depende de la fracción de lixiviación, si la fracción de lixiviación es mayor de 0.45 se mantiene un bajo el nivel de salinidad en la solución del suelo (Hoffman 1990). A medida que la fracción de lixiviación disminuye, se incrementa la salinidad del agua del suelo debido al efecto de concentración. Lo que representa un riesgo de salinidad y toxicidad para los cultivos sensibles a la salinidad $\left(\mathrm{CE}<1 \mathrm{dS} \mathrm{m}^{-1}\right)$ y al boro $\left(<1 \mathrm{mg} \mathrm{L}^{-1}\right.$ de $\mathrm{B}^{3+}$ ) como el frijol, brócoli y trigo (Maas 1990), que son ampliamente cultivados en la zona agrícola estudiada (Figura 4).

La utilización del agua de riego puede restringirse debido al riesgo de toxicidad por cloruro. Cuando la concentración de $\mathrm{Cl}^{-}$es mayor de 10 meq $\mathrm{L}^{-1}$ no se recomienda su uso en la irrigación. Si la concentración de $\mathrm{Cl}^{-}$se encuentra entre 4 y 10 meq $\mathrm{L}^{-1}$ la restricción de uso es moderada (Ayers y Westcot 1987). En este estudio el $95 \%$ de las muestras de agua tuvieron valores bajos de concentración de 


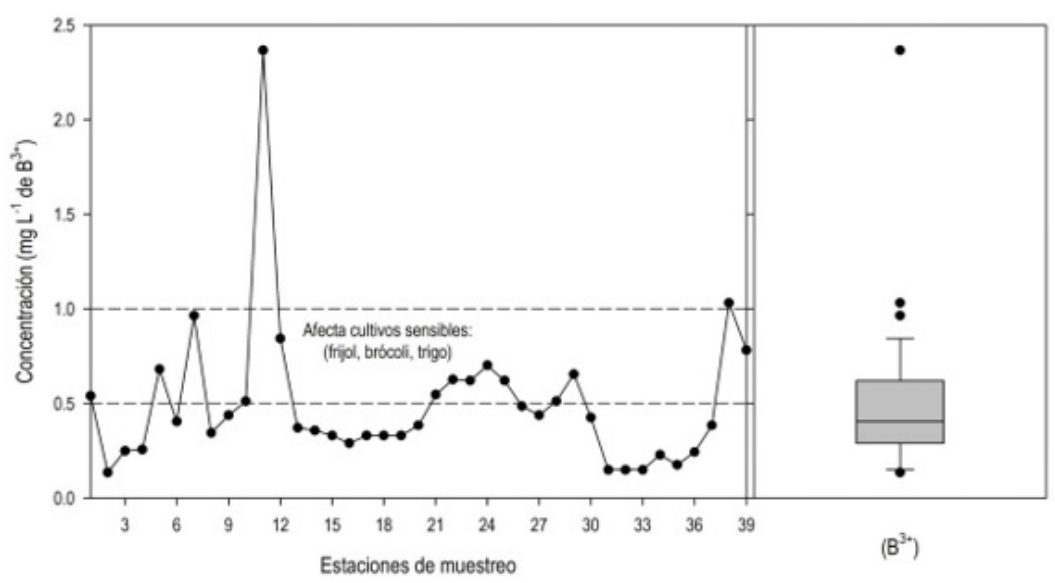

Figura 4. Distribución de la concentración de boro en el agua del río Lerma.

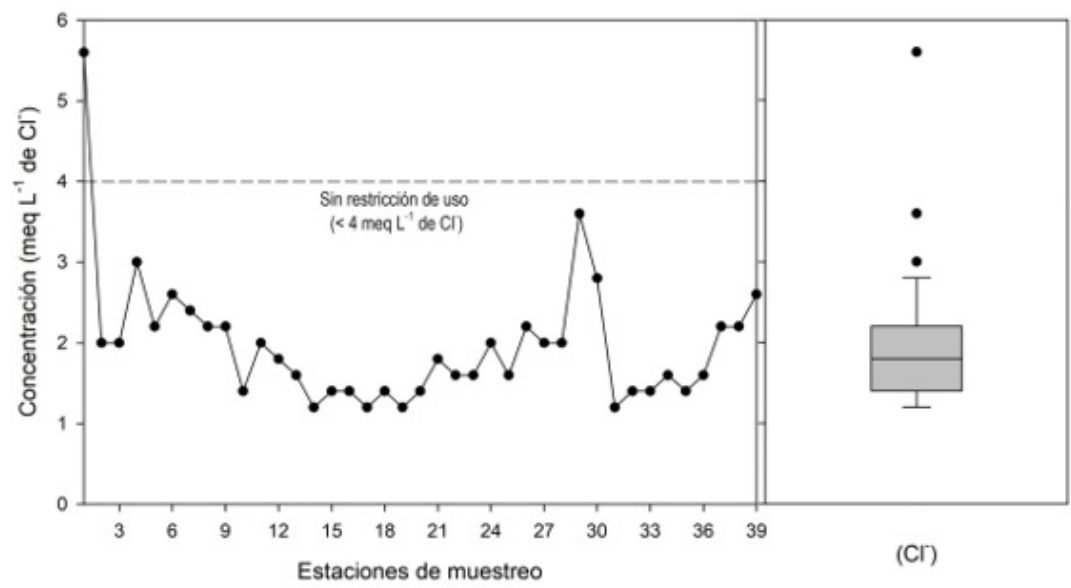

Figura 5. Distribución de la concentración de cloruro en el agua del río Lerma.

$\mathrm{Cl}^{-}$, por lo cual, no presentaron ninguna restricción de uso para este ión (Figura 5).

La concentración de nitrato y fosfato en el agua del río Lerma es elevada, posiblemente tienen origen en las descargas de aguas residuales de tipo doméstico, industrial o agrícola. La elevada concentración de iones representa un riesgo por la posible eutrofización de los cuerpos de agua, además del riesgo de contaminación del agua subterránea por lixiviación de $\mathrm{NO}_{3-}$. El $\mathrm{B}^{3+}$ representa riesgo de toxicidad para los cultivos sensibles, mientras que el contenido de $\mathrm{Cl}^{-}$no presentó restricción de uso agrícola. Por la concentración de nutrientes, en el curso alto del río Lerma el agua puede clasificarse en estado hipereutrófico y en los cursos medio y bajo varía entre hipereutrófico y eutrófico.

\section{LITERATURA CITADA}

Ayers RS, Westcot DW (1987) Water quality for agriculture. FAO, irrigation and drainage, paper 29. Food and Agriculture Organization of the United Nations. Roma. 174p.

Belaid N, Neel C, Kallel M, Ayoub T, Ayadi A, Baudu M (2012) Long term effects of treated wastewater irrigation 
on calcisol fertility: A case study of Sfax-Tunisia. Agricultural Sciences 3: 702-713.

Can-Chulim A, Cruz-Crespo E, Ortega-Escobar HM, Sánchez-Bernal El, Madueño-Molina A, Bojórquez-Serrano $\mathrm{Jl}$, et al. (2017) Respuesta de Phaseolus vulgaris a la salinidad generada por $\mathrm{NaCl}, \mathrm{Na}_{2} \mathrm{SO}_{4}$ y $\mathrm{NaHCO}_{3}$. Revista Mexicana de Ciencias Agrícolas 8: 1287-1300.

Can-Chulim A, Ramírez-Guerrero LG, Ortega-Escobar HM, Cruz-Crespo E, Flores-Román D, Sánchez-Bernal El, et al. (2014) Germination and seedling growth of Phaseolus vulgaris L. in saline conditions. Revista Mexicana de Ciencias Agrícolas 5: 753-763.

Chávez-Alcántar A, Velázquez-Machuca M, Pimentel-Equihua JL, Venegas-González J, Montañez-Soto JL, Vázquez-Gálvez G (2011) Hydrochemistry of surface water in the cienega of Chapala and water quality index. Terra Latinoamericana 9: 83-94.

Cifuentes E, Gómez M, Blumenthal U, Téllez-Rojo MM, Romieu I, Ruíz-Palacios G, et al. (2000) Risk factors for Giardia intestinalis infection in agricultural villages practicing wastewater irrigation in Mexico. The American Journal of Tropical Medicine and Hygiene 62: 388-392.

Cotler-Avalos H, Mazari-Hiriart M, De anda Sánchez J (2006) Atlas de la cuenca Lerma-Chapala: construyendo una visión conjunta. INE-SEMARNAT, México. 196p.

CONAGUA (2015) Estadísticas agrícolas de los distritos de riego. Secretaría del Medio Ambiente y Recursos Naturales, Comisión Nacional del Agua. Tlalpan, México. 390p.

Duncan M, Cairncross S (1990) Directrices para el uso sin riesgos de aguas residuales y excretas en agricultura y acuicultura, medidas de protección de la salud pública. Organización Mundial de la Salud, Ginebra. 213p.

Eaton AD, Greenberg AE, Clescerl SL (1998) Standard methods for the examination of water and wastewater. 20th edition. American Public Health Association, American Water Works Association, Water Pollution Control Federation. Washington, United States of America. 1325p.

Figueruelo-Alejano JE, Marino-Dávila M (2004) Química física del ambiente y de los procesos medioambientales. Editorial Reverté, España. 591p.

Hem JD (1985) Study and interpretation of the chemical characteristics of natural water. Water-supply paper 2254, third edition. U.S. Geological Survey. United States of America. 263p.

Hoffman GJ (1990) Leaching fraction and root zone salinity control. In: Tanji KK (ed). Agricultural salinity assessment and management. ASCE, New York. United States of America. pp. 237-261.

López-García AD, Ortega-Escobar HM, Ramírez-Ayala C, Sánchez-Bernal E, Can-Chulim A, Gómez-Meléndez DJ, Vázquez-Alvarado RE (2016) Caracterización fisicoquímica del agua residual urbano-industrial y su importancia en la agricultura. Tecnología y Ciencias del Agua 7: 139-157.

López-Hernández M, Ramos-Espinoza MG, Carranza-Fraser J (2007) Análisis multimétrico para evaluar contaminación en el río Lerma y lago de Chapala, México. Hidrobiológica 17: 17-30.

Maas EV (1990) Crop salt tolerance. In: Tanji KK (ed). Agricultural salinity assessment and management. ASCE, New York. United States of America. pp: 262-304.

Mancilla-Villa OR, Bautista-Olivas AL, Ortega-Escobar HM, Ramírez-Ayala C, Reyes-Ortigoza AL, Flores-Magdaleno $\mathrm{H}$, et al. (2014) Contenido de boro en el agua superficial de Puebla, Tlaxcala y Veracruz. Tecnología y Ciencias de Agua 5: 97-109

Montgomery DC, Runger GC (2015) Probabilidad y estadística aplicadas a la ingeniería. Segunda edición. Limusa. México. 817p. 
Moreno-Franco DP, Quintero-Manzano J, López-Cuevas A (2010) Métodos para identificar, diagnosticar y evaluar el grado de eutrofia. ContactoS 78: 25-33.

Page AL, Chang AC, Adriano DC (1990) Deficiencies and toxicities of trace elements. In: Tanji KK (ed). Agricultural salinity assessment and management. ASCE, New York. United States of America. pp: 138-160.

Pérez-Díaz JP, Ortega-Escobar HM, Can-Chulim A, Sánchez-Bernal El, Ramírez-Ayala C, Uscanga-Mortera E (2015) Hydrochemical composition and irrigation water quality of Lerma-Chapala river system, Mexico. International Journal of Research and Reviews in Applied Sciences 22: 18-26.

Pratt PF, Suarez DL (1990) Irrigation water quality assessments. In: Tanji KK (ed). Agricultural salinity assessment and management. ASCE, New York. United States of America. pp: 220-236.

Richards LA, Allison LE, Brown JW, Hayward HE, Bernstein L, Fireman M, et al. (1982) Diagnóstico y rehabilitación de suelos salinos y sódicos. Limusa. México. 172p.

Robarge WP, Edwards A, Johnson B (1983) Water and wastewater analysis for nitrate via nitration of salicylic acid. Communications in Soil Science and Plant Analysis 14: 1207-1215.

Robledo-Zacarías VH, Velázquez-Machuca MA, Montañez-Soto JL, Pimentel-Equihua JL, Vallejo-Cardona AA, López-Calvillo MD, et al. (2017) Hidroquímica y contaminantes emergentes en aguas residuales urbano industriales de Morelia, Michoacán, México. Revista Internacional de Contaminación Ambiental 33: 221235.

Rodier J (1978) Análisis de las aguas: aguas naturales, aguas residuales, agua de mar. Ediciones Omega, Barcelona, España. 1057p.

Sánchez-Bernal El, Camacho-Escobar MA, Rodríguez-León A, Ortega-Escobar HM (2013) Physiological behavior of potato cv. Tollocan at diverse types of salinity. Journal of Plant Studies 2: 120-134.

Sánchez-Bernal El, Ortega-Escobar HM, González-Hernández V, Camacho-Escobar M, Kohashi-Shibata J (2008) Crecimiento de plantas de papa (Solanum Tuberosum L.) cv. Alpha, inducido por diversas soluciones salinas. Interciencia 33: 643-650.

SECOFI (1980) NMX-AA-003-SCFI-1980. Aguas residuales, Muestreo. Secretaría de Comercio y Fomento Industrial. Publicada en el Diario Oficial de la Federación. 25 de marzo de 1980.

Velázquez-Machuca MA, Pimentel-Equihua JL, Ortega-Escobar HM (2010) Longitudinal and temporal hydrochemical variations in the Duero River. Agrociencia 44: 599-609.

Velázquez MA, Pimentel JL, Ortega M (2011) Estudio de la distribución de boro en fuentes de agua de la cuenca del río Duero, México, utilizando análisis estadístico multivariado. Revista Internacional de Contaminación Ambiental 27: 19-30.

Zamora F, Rodríguez N, Torres D, Yendis H (2008) Efecto del riego con aguas residuales sobre propiedades químicas de suelos de la planicie de Coro, Estado Falcón. Bioagro 20: 193-199.

Zarazúa G, Ávila-Pérez P, Tejeda S, Valdivia-Barrientos M, Zepeda-Gómez C, Macedo-Miranda G (2013) Evaluación de los metales pesados $\mathrm{Cr}, \mathrm{Mn}, \mathrm{Fe}, \mathrm{Cu}, \mathrm{Zn}$ y $\mathrm{Pb}$ en sombrerillo de agua (Hydrocotyle ranunculoides) del curso alto del río Lerma, México. Revista Internacional de Contaminación Ambiental 29: 17-24. 